\title{
Major Constituents and Cytotoxic Effects of Ajuga chamaecistus ssp. tomentella
}

Nargess Sadati ${ }^{\mathrm{a}}$, Kristina Jenett-Siems ${ }^{\mathrm{b}}$, Karsten Siems ${ }^{\mathrm{c}}$, Mohammad Reza Shams Ardekani $^{\mathrm{a}}$, Abbas Hadjiakhoondi ${ }^{\mathrm{a}}$, Tahmineh Akbarzadeh ${ }^{\mathrm{d}}$, Seyed Nasser Ostad ${ }^{\mathrm{e}}$, and Mahnaz Khanavi ${ }^{\mathrm{a}, *}$

a Department of Pharmacognosy and Medicinal Plants Research Center, Faculty of Pharmacy, Tehran University of Medical Sciences, Tehran 14155-6451, Iran.

Fax:+98 21 66954706. E-mail: khanavim@sina.tums.ac.ir

b Institut für Pharmazie (Pharmazeutische Biologie), Freie Universität Berlin, Berlin, Germany

c Analyticon Discovery GmbH, Potsdam, Germany

d Department of Medicinal Chemistry, Faculty of Pharmacy, Tehran University of Medical Sciences, Tehran 14155-6451, Iran

e Department of Toxicology and Pharmacology and Pharmaceutical Science Research Center, Faculty of Pharmacy, Tehran University of Medical Sciences,

Tehran 14155-6451, Iran

* Author for correspondence and reprint requests

Z. Naturforsch. 67 c, 275-281 (2012); received May 29, 2011/January 8, 2012

The $n$-butanolic fraction of a methanolic extract ( $80 \%$ ) from aerial parts of Ajuga chamaecistus ssp. tomentella was analysed using different chromatographic methods. Column (CC) and high-performance liquid chromatography (HPLC) were used for isolation and purification. ${ }^{13} \mathrm{C}$, ${ }^{1} \mathrm{H}$ NMR, H-H COSY, HSQC, HMBC, and ESI-MS were employed for identification of the compounds isolated from this fraction. The structures of the compounds were determined to be cis-melilotoside (1), trans-melilotoside (2), lavandulifolioside (3), 20-hydroxyecdysone (4), leonoside B (5), martynoside (6), ajugalactone (7), makisterone A (8), and 24-dehydroprecyasterone (9). This is the first report on the presence of cis- and transmelilotoside in Ajuga species. Cytotoxic evaluation of the $n$-butanolic fraction, cis- and transmelilotoside against cancer (T47D, HT-29, and Caco-2) and normal (NIH 3T3) cell lines by the mitochondrial tetrazolium test (MTT) showed no cytotoxic effects up to $400 \mu \mathrm{g} / \mathrm{mL}$. The results of this study suggest that melilotoside, phenylethyl glycosides, and phytoecdysteroids are the main constituents of the $n$-butanolic fraction of Ajuga chamaecistus ssp. tomentella.

Key words: Ajuga chamaecistus ssp. tomentella, Melilotoside, Cytotoxic Effect

\section{Introduction}

More than one hundred species including fifty varieties and subspecies of Ajuga (Lamiaceae) are distributed throughout the world. The genus Ajuga with the common name bugle is found in China, Korea, Japan, and throughout Europe. Five species of this annual and perennial genus are found in Iran, of which Ajuga chamaecistus contains several exclusive subspecies, including $A$. chamaecistus ssp. tomentella (Mozaffarian, 2007). Some species belonging to this genus are used in traditional medicine of different countries of the world, including Iran, for treatment of joints pains, gout, and jaundice (Naghibi et al., 2005). A broad range of biological effects has been reported from different species of Ajuga such as hypoglycemic (Hilaly and Lyoussi, 2002), treatment of joint disease (Ono et al., 2008), anti-inflammatory (Gautam et al., 2011), and antimalarial (Kuria et al., 2001). Many phytochemical studies on Ajuga species were performed which led to the isolation of phytoecdysteroids (Vanyolos et al., 2009; Castro et al., 2008), diterpenoids (Coll, 2002), iridoids (Manguro et al., 2007), and phenylethyl glycosides (Akbay et al., 2003). The aim of the present study was a phytochemical investigation of the $n$-butanolic fraction obtained from aerial parts of Ajuga chamaecistus ssp. tomentella, collected in Tehran (Iran), which has not been previously reported. Furthermore, we examined the cytotoxicity of the $n$-butanolic fraction and two major constituents, isolated from this fraction, against cancer and normal cell lines (T47D, Caco2, HT-29, and NIH 3T3) by the MTT assay. 


\section{Material and Methods}

\section{General experimental procedures}

${ }^{1} \mathrm{H}$ and ${ }^{13} \mathrm{C}$ NMR spectroscopy of compounds 1 and 2 were performed in $\mathrm{CD}_{3} \mathrm{OD}$ on a Bruker Avance DPX 400 spectrometer (Karlsruhe, Germany) [400 MHz, tetramethylsilane (TMS) as internal standard]. ${ }^{1} \mathrm{H}$ and ${ }^{13} \mathrm{C}$ NMR spectra of compounds 3-9 were acquired in $\mathrm{CD}_{3} \mathrm{OD}$ on a Jeol ECX-400 spectrometer (Peabody, MA, USA) (400 MHz, TMS as internal standard). ${ }^{1} \mathrm{H}-$ ${ }^{1} \mathrm{H}$ COSY, HMBC, and HSQC spectra were obtained on a Bruker DRX $500 \mathrm{MHz}$ spectrometer. ESI-mass spectra were recorded on an Agilent 6210 ESI-TOP spectrometer (Santa Clara, CA, USA). Column chromatography (CC) was performed using Sephadex LH-20 $(45 \times 4.5 \mathrm{~cm}$, lipophilic Sephadex, 25-100 $\mu$ m; Sigma, Dorset, UK) and RP-18 $(30 \times 4.5 \mathrm{~cm}$, Lichroprep RP-18, 40-63 $\mu \mathrm{m}$; Merck, Darmstadt, Germany) columns. Analytical and preparative high-performance liquid chromatography (HPLC) separations were performed on a Shimadzu LC-10AD pumping system (Kyoto, Japan) with a Shimadzu variable wavelength detector $(220 \mathrm{~nm})$ equipped with a Knauer (Berlin, Germany) Eurospher 100 C-18 $(7 \mu \mathrm{m}, 250 \times 4 \mathrm{~mm})$ and Nucleosil 300-C18 $(10 \mu \mathrm{m}, 250 \times 16 \mathrm{~mm})$ column, respectively.

\section{Plant material}

Aerial parts of Ajuga chamaecistus Ging. ssp. tomentella (Boiss.) Rech. f. were collected from "Sorkhe Hesar", east of Tehran, Iran, in June 2008 and verified by Prof. G. Amin. A voucher specimen (THE-6697) has been deposited in the herbarium of the Department of Pharmacognosy, Faculty of Pharmacy, Tehran University of Medical Sciences, Tehran, Iran.

\section{Extraction and isolation}

The air-dried and ground plants of $A$. chamaecistus ssp. tomentella $(1 \mathrm{~kg})$ were extracted with $80 \%$ methanol $(7 \times 2.5 \mathrm{~L})$ at room temperature and concentrated under reduced pressure to give a dark brown extract $(180 \mathrm{~g})$. The extract $(150 \mathrm{~g})$ was defatted through repeated extraction with $n$ hexane. The defatted extract was partitioned successively between $80 \%$ methanol, diethyl ether, and $n$-butanol. Twenty g of the $n$-butanolic fraction were loaded on a Sephadex LH-20 (150 g) column and eluted with aqueous methanol $(60 \%)$ to afford 4 fractions. Fraction $2(10 \mathrm{~g})$ was chromatographed on an RP-18 column, under medium pressure, and eluted with a gradient of water and methanol $(80: 20-50: 50 \mathrm{v} / \mathrm{v})$ to afford 5 fractions $(\mathrm{B}-\mathrm{F})$. Fraction B $(8 \mathrm{~g})$ was rechromatographed on RP-18 material with a gradient of aqueous methanol $(5 \%, 20 \%$, and $50 \%)$ to give 3 fractions $\left(\mathrm{B}_{1}-\mathrm{B}_{3}\right) . \mathrm{B}_{1}(200 \mathrm{mg})$ was subjected to semipreparative reversed phase (RP)-HPLC using gradient elution with aqueous methanol $(10 \%-35 \%$; flow-rate, $1 \mathrm{~mL} / \mathrm{min}$; time per run, $30 \mathrm{~min}$ ) to afford compound $\mathbf{1}(14 \mathrm{mg})$. Compound $2(20 \mathrm{mg})$ was obtained by preparative RP-HPLC and a gradient of aqueous methanol $(30 \%-35 \%)$ within $40 \mathrm{~min}$ from fraction $\mathrm{B}_{2}(190 \mathrm{mg})$. Fraction $\mathrm{B}_{3}(2.5 \mathrm{~g})$ was chromatographed on an RP-18 column eluted with a gradient of water/methanol $(80: 20-50: 50 \mathrm{v} / \mathrm{v})$ to give 9 fractions $\left(\mathrm{B}_{3.1}-\mathrm{B}_{3.9}\right)$. Purification of $B_{3.2}(200 \mathrm{mg})$ using preparative RP-HPLC with a gradient of aqueous methanol $(30 \%-60 \%$; flow-rate, $7 \mathrm{~mL} / \mathrm{min})$ within $60 \mathrm{~min}$ afforded compound $\mathbf{3}(5 \mathrm{mg})$ and compound $\mathbf{4}$ $(80 \mathrm{mg})$. Fraction $\mathrm{B}_{3.7}(144 \mathrm{mg})$ was subjected to RP-HPLC with aqueous methanol $(35 \%-60 \%)$ to give compounds $5(13.5 \mathrm{mg})$ and $\mathbf{6}(17.6 \mathrm{mg})$ within $60 \mathrm{~min}$. Fraction C ( $200 \mathrm{mg})$ was purified by RP-HPLC with a gradient of aqueous methanol $(30 \%-60 \%)$ within 60 min to obtain compound $7(8 \mathrm{mg})$ and compound $6(11.5 \mathrm{mg})$. Fraction C (100 mg) was further purified by RP-HPLC using a gradient of water/acetonitrile (80:20-65:35) to afford compound $8(1 \mathrm{mg})$ and compound 9 $(1 \mathrm{mg})$. Direct sunlight was excluded during extraction and purification of the compounds.

cis-Melilotoside (1): ${ }^{1} \mathrm{H} \quad$ NMR $\quad(400 \mathrm{MHz}$, $\left.\mathrm{CD}_{3} \mathrm{OD}\right): \delta=7.52(1 \mathrm{H}, d, J=7.46 \mathrm{~Hz}, \mathrm{H}-6)$, $7.31(1 \mathrm{H}, d, J=12.52 \mathrm{~Hz}, \mathrm{H}-7), 7.28(1 \mathrm{H}, d d$, $J=8.561 .24, \mathrm{~Hz}, \mathrm{H}-4), 7.18(1 \mathrm{H}, d, J=8.28 \mathrm{~Hz}$, $\mathrm{H}-3), 6.95(1 \mathrm{H}, t, J=7.47 \mathrm{~Hz}, \mathrm{H}-5), 5.95(1 \mathrm{H}, d$, $J=12.52 \mathrm{~Hz}, \mathrm{H}-8), 4.93(1 \mathrm{H}, d, J=7.24 \mathrm{~Hz}$, GluH-1'), 3.87 (1H, Ha-6'), 3.68 (1H, Hb-6'), 3.47 (1H, H-3'), 3.45 (1H, H-2'), 3.40 (2H, H-4', H-5'). $-{ }^{13} \mathrm{C}$ NMR $\left(\mathrm{CD}_{3} \mathrm{OD}\right): \delta=138.42(\mathrm{C}-7), 130.48(\mathrm{C}-$ 4), 130.27 (C-6), 121.64 (C-5), 120.02 (C-8), 115.27 (C-3); glucose: 101.43 (C-1'), 77.04 (C-5'), 76.95 (C-3'), 73.76 (C-2'), 70.00 (C-4'), 61.32 (C-6'). ESI-TOF-MS (positive): $\mathrm{m} / z=349.08[\mathrm{M}+\mathrm{Na}]^{+}$, $365.05[\mathrm{M}]^{+}$.

trans-Melilotoside (2): ${ }^{1} \mathrm{H} \quad \mathrm{NMR} \quad(400 \mathrm{MHz}$, $\left.\mathrm{CD}_{3} \mathrm{OD}\right): \delta=8.14(1 \mathrm{H}, d, J=16.20 \mathrm{~Hz}, \mathrm{H}-7), 7.64$ $(1 \mathrm{H}, d, J=7.68 \mathrm{~Hz}, \mathrm{H}-6), 7.42(1 \mathrm{H}, m, \mathrm{H}-4), 7.26$ 
$(1 \mathrm{H}, m, \mathrm{H}-3), 7.07(1 \mathrm{H}, t, J=7.48 \mathrm{~Hz}, \mathrm{H}-5), 6.54$ $(1 \mathrm{H}, d, J=16.2 \mathrm{~Hz}, \mathrm{H}-8), 5.00(1 \mathrm{H}, d, J=7.68 \mathrm{~Hz}$, Glu-H-1'). - ESI-TOF-MS (positive): $m / z=349.08$ $[\mathrm{M}+\mathrm{Na}]^{+}, 365.05[\mathrm{M}]^{+}$.

Lavandulifolioside (3): ${ }^{1} \mathrm{H}$ NMR $(400 \mathrm{MHz}$, $\mathrm{CD}_{3} \mathrm{OD}$ ): see Table I. - ESI-TOF-MS (positive): $m / z=779.2384[\mathrm{M}+\mathrm{Na}]^{+}$.

20-Hydroxyecdysone (4): ${ }^{1} \mathrm{H}$ NMR $(400 \mathrm{MHz}$, $\left.\mathrm{CD}_{3} \mathrm{OD}\right): \delta=5.79(1 \mathrm{H}, d, J=2.3 \mathrm{~Hz}, \mathrm{H}-7), 3.95$ $\left(1 \mathrm{H}, b r s, \mathrm{H}_{\mathrm{eq}}-3\right), 3.82\left(1 \mathrm{H}, m, \mathrm{H}_{\mathrm{ax}}-2\right), 3.13(1 \mathrm{H}$, $b r t, \mathrm{H}-9), 2.37$ (1H, dd, H-5), 1.19 (6H, s, Me26,27), 1.18 (3H, s, Me-21), 0.94 (3H, s, Me-19), 0.86 (3H, $s, \mathrm{Me}-18)$. - ESI-TOF-MS (positive): $m / z=503.298[\mathrm{M}+\mathrm{Na}]^{+}, 983.607\left[\mathrm{M}_{2}+\mathrm{Na}\right]^{+}$.

Leonoside B (5): ${ }^{1} \mathrm{H}$ NMR (400 $\left.\mathrm{MHz}, \mathrm{CD}_{3} \mathrm{OD}\right)$ : see Table I. - ESI-TOF-MS (positive): $\mathrm{m} / \mathrm{z}=$ $807.27[\mathrm{M}+\mathrm{Na}]^{+}, 823.24[\mathrm{M}+\mathrm{K}]^{+}$.
Martynoside (6): ${ }^{1} \mathrm{H}$ NMR (400 $\left.\mathrm{MHz}, \mathrm{CD}_{3} \mathrm{OD}\right)$ : see Table I. - ESI-TOF-MS (positive): $\mathrm{m} / \mathrm{z}=$ $675.23[\mathrm{M}+\mathrm{Na}]^{+}, 691.20[\mathrm{M}+\mathrm{K}]^{+}$.

Ajugalactone (7): This is the first report on NMR data of ajugalactone achieved in $\mathrm{CD}_{3} \mathrm{OD}$. ${ }^{1} \mathrm{H}$ NMR (400 MHz, CD $\left.\mathrm{CD}_{3} \mathrm{OD}\right): \delta=5.95(1 \mathrm{H}$, $J=2.56 \mathrm{~Hz}, \mathrm{H}-7), 4.19(1 \mathrm{H}, d d, J=12.96,4.16 \mathrm{~Hz}$, $\mathrm{H}-22), 3.95(1 \mathrm{H}, b r s, \mathrm{H}-3), 3.79(1 \mathrm{H}, m, \mathrm{H}-2), 3.55$ $(1 \mathrm{H}, b r t, J=10.9 \mathrm{~Hz}, \mathrm{H}-9), 2.92(1 \mathrm{H}, b r t, J=$ $10.2 \mathrm{~Hz}, \mathrm{H}-17), 2.79\left(1 \mathrm{H}, q, \mathrm{H}_{\mathrm{a}}-11\right), 2.38-2.32$ $(2 \mathrm{H}, m, \mathrm{H}-23,28), 2.10-2.04\left(1 \mathrm{H}, m, \mathrm{H}_{\mathrm{a}}-16\right)$, $1.76-1.72\left(1 \mathrm{H}, m, \mathrm{H}_{\mathrm{b}}-16\right), 2.23-2.17(1 \mathrm{H}, m$, $\left.\mathrm{H}_{\mathrm{a}}-15\right), 1.85$ (3H, br s, Me-18), $1.76-1.72(1 \mathrm{H}, m$, $\left.\mathrm{H}_{\mathrm{b}}-15\right), 1.64\left(1 \mathrm{H}, d, J=4.4 \mathrm{~Hz}, \mathrm{H}_{\mathrm{a}}-1\right), 1.47(1 \mathrm{H}, b r$ $\left.s, \mathrm{H}_{\mathrm{b}}-1\right), 1.25$ (3H, s, Me-26), 1.21 (3H, $\left.s, \mathrm{Me}-18\right)$, $1.12(3 \mathrm{H}, t, J=7.64 \mathrm{~Hz}, \mathrm{Me}-29), 1.07$ (3H, $s, \mathrm{Me}-$ 19). $-{ }^{13} \mathrm{C}$ NMR $\left(500 \mathrm{MHz}, \mathrm{CD}_{3} \mathrm{OD}\right): \delta=202.1$ (C-6), 123.7 (C-7), 162.2 (C-8), 210.2 (C-12), 89.4

Table I. ${ }^{1} \mathrm{H}$ NMR spectral data of $\mathbf{3}, \mathbf{5}$, and $\mathbf{6}^{\mathrm{a}}$.

\begin{tabular}{|c|c|c|c|c|}
\hline $\mathrm{H}$ & & 3 & 5 & 6 \\
\hline \multirow[t]{7}{*}{ Aglycone } & $\mathrm{H}-\mathrm{C}(2)$ & $6.68(d, J=2.08)$ & $6.72(d, J=2.08)$ & $6.71(d, J=2.08)$ \\
\hline & $\mathrm{H}-\mathrm{C}(5)$ & $6.66(d, J=7.92)$ & $6.81(d, J=4.64)$ & $6.81(d, J=4.4)$ \\
\hline & $\mathrm{H}-\mathrm{C}(6)$ & $6.56(d d, J=8.12,2.08)$ & $6.68(d d, J=8.12,2.08)$ & $6.68(d, J=8.12,2.12)$ \\
\hline & $\mathrm{CH}_{2}(\alpha)$ & $4.04,3.77-3.23$ & $4.08,3.73(\mathrm{~m})$ & $4.07,3.77-3.35(m)$ \\
\hline & $\mathrm{CH}_{2}(\beta)$ & $2.79(b r t, J=6.96)$ & $2.82(b r t, J=7.44)$ & $2.82(b r t, J=7.4)$ \\
\hline & $\mathrm{CH}_{3} \mathrm{O}$ & - & $3.86(s)$ & $3.86(s)$ \\
\hline & $\mathrm{CH}_{3} \mathrm{O}$ & - & $3.79(s)$ & $3.79(\mathrm{~s})$ \\
\hline \multirow[t]{7}{*}{$\beta$-Glucose } & $\mathrm{H}-\mathrm{C}\left(1^{\prime}\right)$ & $4.36(d, J=7.92)$ & $4.37(d, J=8.12)$ & $4.37(d, J=8.12)$ \\
\hline & $\mathrm{H}-\mathrm{C}\left(2^{\prime}\right)$ & $3.77-3.23$ & $3.70-3.47$ & $3.77-3.35$ \\
\hline & $\mathrm{H}-\mathrm{C}\left(3^{\prime}\right)$ & $3.77-3.23$ & $3.70-3.47$ & $3.77-3.35$ \\
\hline & $\mathrm{H}-\mathrm{C}\left(4^{\prime}\right)$ & $4.92(t)$ & $4.94(t)$ & $4.92(t)$ \\
\hline & $\mathrm{H}-\mathrm{C}\left(5^{\prime}\right)$ & $3.77-3.23$ & $3.70-3.47$ & $3.77-3.35$ \\
\hline & $\mathrm{H}-\mathrm{C}\left(6_{\mathrm{A}}^{\prime}\right)$ & $3.77-3.23$ & $3.70-3.47$ & $3.77-3.35$ \\
\hline & $\mathrm{H}-\mathrm{C}\left(6_{\mathrm{B}}^{\prime}\right)$ & $3.85(d d, J=12.96,2.4)$ & 3.85 & $3.77-3.35$ \\
\hline \multirow[t]{6}{*}{$\alpha$-Rhamnose } & $\mathrm{H}-\mathrm{C}\left(1^{\prime \prime}\right)$ & $5.46(b r s)$ & $5.47(d, J=1.4)$ & $5.18(d, J=1.6)$ \\
\hline & $\mathrm{H}-\mathrm{C}\left(2^{\prime \prime}\right)$ & $3.92(d d, J=3.24,1.36)$ & $3.93(b r s)$ & $3.92(b r s)$ \\
\hline & $\mathrm{H}-\mathrm{C}\left(3^{\prime \prime}\right)$ & $3.77-3.23$ & $3.70-3.47$ & $3.77-3.35$ \\
\hline & $\mathrm{H}-\mathrm{C}\left(4^{\prime \prime}\right)$ & $3.77-3.23$ & $3.70-3.47$ & $3.77-3.35$ \\
\hline & $\mathrm{H}-\mathrm{C}\left(5^{\prime \prime}\right)$ & $3.77-3.23$ & $3.70-3.47$ & $3.77-3.35$ \\
\hline & $\mathrm{CH}_{3}(6 ”)$ & $1.05(d, J=6.28)$ & $1.05(d, J=6.28)$ & $1.08(d, J=6.24)$ \\
\hline \multirow[t]{5}{*}{$\alpha$-Arabinose } & $\mathrm{H}-\mathrm{C}(1 ”)$ & $4.29(d, J=7.2)$ & $4.30(d, J=7.44)$ & - \\
\hline & $\mathrm{H}-\mathrm{C}(2 ")$ & $3.77-3.23$ & $3.70-3.47$ & - \\
\hline & $\mathrm{H}-\mathrm{C}(3 ")$ & $3.77-3.23$ & $3.70-3.47$ & - \\
\hline & $\mathrm{H}-\mathrm{C}(4 ")$ & $3.77-3.23$ & $3.75(b r s)$ & - \\
\hline & $\mathrm{H}-\mathrm{C}\left(5^{\prime \prime}\right)$ & $3.77-3.23$ & $3.70-3.47$ & - \\
\hline \multirow[t]{5}{*}{ Caffeic acid } & $\mathrm{H}-\mathrm{C}\left(2{ }^{\prime \prime \prime}\right)$ & $7.03(d, J=1.84)$ & $7.18(d, J=1.8)$ & $7.18(d, \bar{J}=1.84)$ \\
\hline & $\mathrm{H}-\mathrm{C}\left(5^{\prime \prime \prime}\right)$ & $6.76(d, J=8.12)$ & $6.79(d, J=4.64)$ & $6.79(d, J=4.64)$ \\
\hline & $\mathrm{H}-\mathrm{C}\left(6^{\prime \prime \prime}\right)$ & $6.95(d d, J=8.36,2.08)$ & $7.07(d d, J=8.36,1.88)$ & $7.07(d d, J=8.36,1.88)$ \\
\hline & $\mathrm{H}-\mathrm{C}\left(\alpha^{\prime}\right)$ & $6.27(d, J=15.8)$ & $6.37(d, J=16)$ & $6.37(d, J=16)$ \\
\hline & $\mathrm{H}-\mathrm{C}\left(\beta^{\prime}\right)$ & $7.59(d, J=15.88)$ & $7.66(d, J=16)$ & $7.66(d, J=15.76)$ \\
\hline
\end{tabular}

a The spectra were measured in $\mathrm{CD}_{3} \mathrm{OD}(400 \mathrm{MHz})$. Chemical shifts in ppm relative to the internal standard TMS; $J$ in $\mathrm{Hz}$. 
(C-14), 83.3 (C-22), 168.2 (C-27), 154.8 (C-24), 121.5 (C-25). - ESI-TOF-MS (positive): $\mathrm{m} / \mathrm{z}=$ $539.26[\mathrm{M}+\mathrm{Na}]^{+}, 555.23[\mathrm{M}+\mathrm{K}]^{+}, 1055.53\left[\mathrm{M}_{2}+\mathrm{Na}\right]^{+}$.

Makisterone $A \quad(\mathbf{8}):{ }^{1} \mathrm{H} \quad \mathrm{NMR} \quad(400 \mathrm{MHz}$, $\left.\mathrm{CD}_{3} \mathrm{OD}\right): \delta=5.79(1 \mathrm{H}, d, J=2.5 \mathrm{~Hz}, \mathrm{H}-7), 1.17$ $(3 \mathrm{H}, s, \mathrm{Me}-21), 1.14(3 \mathrm{H}, s, \mathrm{Me}-26), 1.11(3 \mathrm{H}$, $s, \mathrm{Me}-27), 0.94(3 \mathrm{H}, s, \mathrm{Me}-19), 0.92(3 \mathrm{H}, d, J=$ $6.72 \mathrm{~Hz}, \mathrm{Me}-28), 0.87$ (3H, $s, \mathrm{Me}-18)$. - ESI-TOFMS (positive): $m / z=517.32[\mathrm{M}+\mathrm{Na}]^{+}$.

24-Dehydroprecyasterone (9): $\quad{ }^{1} \mathrm{H} \quad \mathrm{NMR}$ (400 MHz, $\left.\mathrm{CD}_{3} \mathrm{OD}\right): \delta=5.80(1 \mathrm{H}, d, J=2.3 \mathrm{~Hz}$, $\mathrm{H}-7), 1.83(3 \mathrm{H}, d, J=2.0 \mathrm{~Hz}, \mathrm{Me}-26), 1.31(3 \mathrm{H}$, $s, \mathrm{Me}-21), 1.28(3 \mathrm{H}, d, J=6.4 \mathrm{~Hz}, \mathrm{Me}-19), 0.94$ (3H, $s, \mathrm{Me}-19), 0.87$ (3H, $s, \mathrm{Me}-18)$. - ESI-TOFMS (positive): $m / z=541.28[\mathrm{M}+\mathrm{Na}]^{+}$.

\section{Cell culture}

The colon carcinoma (HT-29), colorectal adenocarcinoma (Caco-2), and breast ductal carcinoma (T47D) cell lines, respectively, were maintained as exponentially growing cultures in RPMI 1640 cell culture medium (PAA, Pasching, Austria) supplemented with $10 \%$ fetal bovine serum (FBS; PAA) for HT-29 cells and 15\% FBS for Caco-2 and T47D cells. The Swiss mouse embryo fibroblast (NIH 3T3) cell line was kept in Dulbecco's modified Eagle's medium (DMEM; PAA) supplemented with $10 \%$ FBS. One hundred IU/mL penicillin and $100 \mu \mathrm{g} / \mathrm{mL}$ streptomycin (Roche, Penzberg, Germany) were added to the media. All cell lines were cultured at $37^{\circ} \mathrm{C}$ in air $/ \mathrm{CO}_{2}(95: 5 \mathrm{v} / \mathrm{v})$ atmosphere.

\section{Determination of cell viability by the MTT assay}

Cytotoxic activities of the $n$-butanolic fraction, cis- and trans-melilotoside from Ajuga chamaecistus ssp. tomentella against breast ductal carcinoma (T47D), colon carcinoma (HT-29), colorectal adenocarcinoma (Caco-2), and Swiss mouse embryo fibroblast (NIH 3T3) cell lines were performed according to our previous study (Khanavi et al., 2010) by the mitochondrial tetrazolium test (MTT).

\section{Results and Discussion}

Isolated compounds 1-9 from the $n$-butanolic fraction of the total methanolic extract of aerial parts of Ajuga chamaecistus ssp. tomentella were identified by comparison of their NMR $\left({ }^{1} \mathrm{H},{ }^{13} \mathrm{C}\right.$
NMR, HMBC, HSQC and ${ }^{1} \mathrm{H}^{-1} \mathrm{H}$ COSY) and ESI-mass spectral data with those reported in the literature.

Compounds $\mathbf{1}$ and $\mathbf{2}$ (Fig. 1) were identified as cis-melilotoside and trans-melilotoside, respectively. This is the first report on the occurrence of cis- and trans-melilotosides in the genus Ajuga. Melilotoside is a coumaric acid derivative which was reported for the first time from Melilotus altissima and $M$. arvensis (Takaishi, 1968). cis-Melilotoside has been reported from several plants (Ferreira and Rodriguezde Oliveira, 2010; Yang et al., 2007a, b), also there is an older report on the occurrence of both cis- and transforms of melilotoside in Melilotus albus depending on growing conditions (Kahnt, 1962). Antiprotozoal activity of melilotoside isolated from Teloxys graveolens, a medicinal plant for treatment of dysentery and diarrhea, has been shown (Calzada et al., 2003).

Compounds 3, 5, and 6 (Fig. 1) were characterized as phenylethanoid glycosides, lavandulifolioside (3), leonoside B (5), and martynoside (6), by comparison of their spectral data with literature values (Basaran et al., 1988; Calis et al., 1992; Sasaki et al., 1978). This group of phenolic compounds has interesting biological properties such as antimicrobial, antibacterial, cytotoxic, antioxidant, enzyme inhibitory, and immunomodulatory. Lavandulifolioside, a trisaccharide phenylethyl glycoside, showed inhibition of peroxylipid formation (Jimenez and Riguera, 1994), a negative chronotropic effect, and decrease of blood pressure (Milkowska-Leyck et al., 2002). Several pharmacological activities of martynoside have been reported, including antioxidant (Miao et al., 2003) and estrogenic/antiestrogenic properties in breast cancer cells (Papoutsi et al., 2006).

The isolated compounds 4 and 7-9 (Fig. 1) were identified as ecdysteroids, 20-hydroxyecdysone (4), ajugalactone (7), makisterone A (8), and 24-dehydroprecyasterone (9) by comparison of their spectral data with data in the literature (Wessner et al., 1992; Imai et al., 1968, 1970). The ecdysteroids are a large class of polyhydroxysteroids isolated from both the animal and plant kingdom. Most of the Ajuga species have been used in traditional medicine all over the world. Several studies have shown that ecdysteroids isolated from Ajuga species are responsible for their biological activities. This group of natural products produces a wide range of pharmacological 
<smiles>COc1ccccc1/C=C/C(=O)O</smiles>

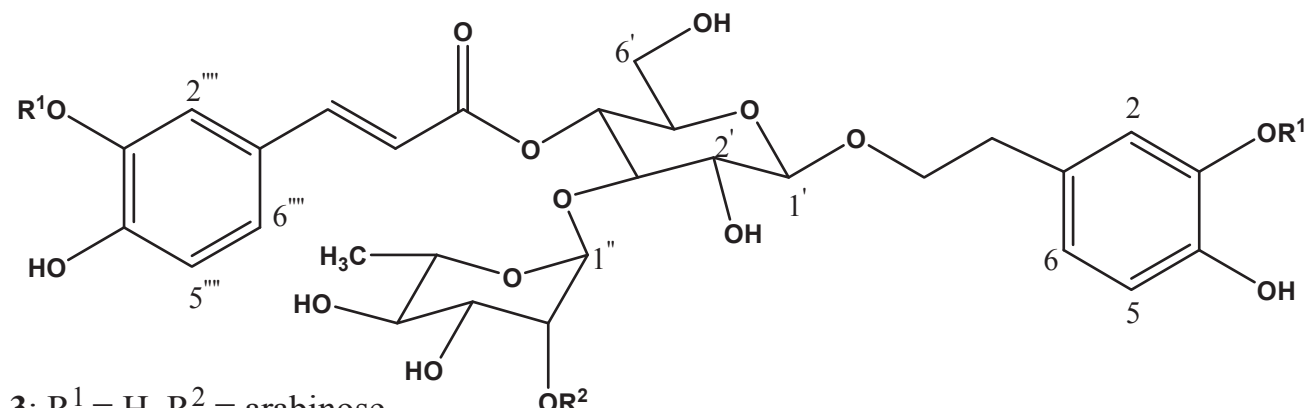

3: $\mathrm{R}^{1}=\mathrm{H}, \mathrm{R}^{2}=$ arabinose

5: $\mathrm{R}^{1}=\mathrm{CH}_{3}, \mathrm{R}^{2}=$ arabinose

6: $\mathrm{R}^{1}=\mathrm{CH}_{3}, \mathrm{R}^{2}=\mathrm{H}$<smiles>C[C@@]12C[C@H](O)[C@@H](O)C[C@H]1C(=O)C=C1CCCCC12</smiles>
se $\mathrm{OR}^{2}$<smiles>CCC1=C(C)C(=O)OCC1</smiles><smiles>CC1=C([C@H](C)O)C[C@@H]([C@](C)(O)[C@H]2CC[C@]3(O)C4=CC(=O)C5C[C@H](O)[C@@H](O)C[C@]5(C)C4CC[C@@]23C)OC1</smiles>

Fig. 1. Molecular structures of cis-melilotoside (1), trans-melilotoside (2), lavandulifolioside (3), 20-hydroxyecdysone (4), leonoside B (5), martynoside (6), ajugalactone (7), makisterone A (8), and 24-dehydroprecyasterone (9), isolated from the $n$-butanolic fraction of Ajuga chamaecistus ssp. tomentella. 
activities in mammals, including adaptogenic and anabolic, and shows increasing protein synthesis in muscles (Gorelick-Feldman et al., 2010), antidiabetic and hypoglycemic (Hamden et al., 2008; Kutepova et al., 2001), hepatoprotective, immunoprotective, wound-healing (Dinan, 2009), antioxidant, and free radical scavenging effects (Cai et al., 2002), and perhaps even antitumour effects (Akbay et al., 2002). 20-Hydroxyecdysone and cyasterone, in addition to ajugalactone, seem to be the most common compounds in Ajuga species (Ramazanov, 2005).

In the cytotoxic evaluation of the $n$-butanolic fraction, cis- (1) and trans-melilotosides (2) did not show cytotoxic effects up to $400 \mu \mathrm{g} / \mathrm{mL}$ against cancer (T47D, HT-29, and Caco-2) and normal (NIH 3T3) cell lines in the MTT assay.

Previous to this study, we isolated three major compounds (20-hydroxyecdysone, cyasterone, and

Akbay P., Gertsch J., Calis I., Heilmann J., Zerbe O., and Sticker O. (2002), Novel antileukemic sterol glycosides from Ajuga salicifolia. Helv. Chim. Acta 85, 1930-1942.

Akbay P., Calis I., Heimann J., and Sticher O. (2003), Ionon, iridoid and phenylethanoid glycosides from Ajuga salicifolia. Z. Naturforsch. 58c, 177-180.

Basaran A. A., Calis I., Anklin C., Nishibe S., and Sticher O. (1988), Lavandulifolioside: A new phenylpropanoid glycoside from Stachys lavandulifolia. Helv. Chim. Acta 71, 1483-1489.

Cai Y.-J., Dai J.-Q., Fang J.-G., Ma L.-P., Hou L.-F., Yang L., and Liu Z.-L. (2002), Antioxidative and free radical scavenging effects of ecdysteroids from Serratula strangulate. Can. J. Physiol. Pharmacol. 80, 1187-1194.

Calis I., Ersoz T., Tasdemir D., and Ruedi P. (1992), Two phenylpropanoid glycosides from Leonurus glaucescens. Phytochemistry 31, 357-359.

Calzada F., Velazquez C., Cedillo-Rivera R., and Esquivel B. (2003), Antiprotozoal activity of the constituents of Teloxys graveolens. Phytother. Res. 17, $731-732$.

Castro A., Coll J., Tandron Y. A., Pant A. K., and Mathela C. (2008), Phytoecdysteroids from Ajuga macrosperma var. breviflora roots. J. Nat. Prod. 71, 1294-1296.

Coll J. (2002), NMR shift data of neo-clerodane diterpenes from the genus Ajuga. Phytochem. Anal. 13, $372-380$.

Dinan L. (2009), Phytoecdysteroids: what use are they? Arch. Insect Biochem. Physiol. 72, 126-141.

Ferreira F. P. and Rodrigues de Oliveira D. C. (2010), New constituents from Mikania laevigata Shultz Bip. ex Baker. Tetrahedron Lett. 51, 6856-6859. 8-acetylharpagide) from the diethyl ether fraction of Ajuga chamaecistus ssp. tomentella, which were inactive in the cytotoxicity evaluation (Sadati et al., 2012)

In conclusion, our study showed that cis- and trans-melilotosides, phenylethyl glycosides, and phytoecdysteroids can be considered major constituents of the $n$-butanolic fraction of Ajuga chamaecistus ssp. tomentella. According to these results, it can be stated that the $n$-butanolic fraction and two major compounds isolated from this fraction are not cytotoxic against cancer and normal cell lines.

\section{Acknowledgements}

This study was part of a $\mathrm{PhD}$ thesis funded and supported by Tehran University of Medical Sciences (TUMS) (grant No. 11302).

Gautam R., Jachak S. M., and Saklani A. (2011), Anti-inflammatory effect of Ajuga bracteosa Wall ex Benth. mediated through cyclooxygenase (COX) inhibition. J. Ethnopharmacol. 133, 928-930.

Gorelick-Feldman J., Cohick W., and Raskin I. (2010), Ecdysteroids elicit a rapid $\mathrm{Ca}^{2+}$ flux leading to Akt activation and increased protein synthesis in skeletal muscle cells. Steroids 75, 632-637.

Hamden K., Ayadi K., Jamoussi K., Masmoudi H., and Eifeki A. (2008), Therapeutic effect of phytoecdysteroids rich extract from Ajuga iva on alloxan induced diabetic rats liver, kidney and pancreas. BioFactors 33, $165-175$.

Hilaly J. and Lyoussi B. (2002), Hypoglycemic effect of lyophilized aqueous extract of Ajuga iva in normal and streptozotocin diabetic rats. J. Ethnopharmacol. 80, $109-113$.

Imai S., Hori M., Fujioka S., Murata E., Goto M., and Nakanishi K. (1968), Isolation of four new phytoecdysones, makisterone A, B, C, D and the structure of makisterone $\mathrm{A}, \mathrm{a}_{28}$ steroid. Tetrahedron Lett., $3883-3886$

Imai S., Murata E., Fujioka S., Matsuoka T., Koreeda M., and Nakanishi K.(1970), Ajugalactone, an insect moulting inhibitor as tested by the chio dipping method. J. Am. Chem. Soc. 92, 7512-7513.

Jimenez C. and Riguera R. (1994), Phenylethanoid glycosides in plants: structure and biological activity. Nat. Prod. Rep. 11, 591-606.

Kahnt G. (1962), Isolierung des trans- und cis-o-Oxyzimtsäureglucosids aus Steinkleeblättern (Melilotus albus) und Umwandlung der trans-Form in die isomere Verbindung in vivo und in vitro durch Sonnenlicht. Naturwissenschaften 49, 207-208. 
Khanavi M., Nabavi M., Sadati N., Shams Ardekani M. R., Sohrabipour J., Nabavi S. M. B., Ghaeli P., and Ostad S. N. (2010), Cytotoxic activity of some marine brown algae against cancer cell lines. Biol. Res. 43, $31-37$.

Kuria K. A. M., Coster S., Muriuki G., Masengo W., Kibwage I., Hoogmartens J., and Laekeman G. M. (2001), Antimalarial activity of Ajuga remota Benth (Labiatae) and Caesalpinia volkensii Harms (Caesalpiniaceae): in vitro confirmation of ethnopharmacological use. J. Ethnopharmacol. 74, 141-148.

Kutepova T. A., Syrov V. N., Khushbaktova Z. A., and Saatov Z. (2001), Hypoglycemic activity of the total ecdysteroid extract from Ajuga turkestanica. Pharm. Chem. J. (Translation of Khimiko-Farmatsevticheskii Zhurnal) 35, 608-609.

Manguro L. O. A., Ogur J. A., Okora D. M., Wagal S. O., and Lemen P. (2007), Further flavonol and iridoid glycosides from Ajuga remota. J. Asian Nat. Prod. Res. 9, 617-629.

Miao J., Wang W., Yao S., Navaratnam S., and Parsons B. J. (2003), Antioxidative properties of martynoside: pulse radiolysis and laser photolysis study. Free Radical Res. 37, 829-833.

Milkowska-Leyck K., Filipek B., and Strezelecka H. (2002), Pharmacological effects of lavandulifolioside from Leonurus cardiac. J. Ethnopharmacol. 80, $85-90$.

Mozaffarian V. (2007), A Dictionary of Iranian Plant Names. Farhang moaser, Tehran, pp. 21-22.

Naghibi F., Mosaddegh M., Mohammadi Motamed S., and Ghorbani A. (2005), Labiatae family in folk medicine in Iran: from ethnobotany to pharmacology. Iran. J. Pharm. Res. 2, 63-79.

Ono Y., Fukaya Y., Imai S., and Yamakuni T. (2008), Beneficial effects of Ajuga decumbens on osteoporosis and arthritis. Biol. Pharm. Bull. 31, 1199-1204.
Papoutsi Z., Kassi E., Mitakou S., Aligiannis N., Tsiapara A., Chrousos G. P., and Moutsatsou P. (2006), Acteoside and martynoside exhibit estrogenic/antiestrogenic properties. J. Steroid Biochem. Mol. Biol. 98, 63-71.

Ramazanov N. Sh. (2005), Phytoecdysteroids and other biologically active compounds from plants of genus Ajuga. Chem. Nat. Compd. 41, 361-369.

Sadati N., Ostad S. N., Karimian Z., Shams Ardekani M. R., Akbarzadeh T., Hadjiakhoondi A., and Khanavi M. (2012), Phytochemical study and in vitro cytotoxic effect of Ajuga chamaecistus ssp. tomentella. Asian J. Chem. 24, 2871-2874.

Sasaki H., Taguchi H., Endo T., Yosioka I., Higashiyama K., and Otomasu H. (1978), The glycosides of Martynia louisiana Mill. A new phenylpropanoid glycoside, martynoside. Chem. Pharm. Bull. 26, 2111-2121.

Takaishi K. (1968), Studies on the coumarin components in the leaves of cherry-tree. Yakugaku Zasshi 88, $1467-1471$.

Wessner H., Champion B., Girault J.-P., Saidi B., and Lafont R. (1992), Ecdysteroids from Ajuga iva. Phytochemistry 31, 3785-3788.

Vanyolos A., Simon A., Toth G., Polgar L., Kele Z., Ilku A., Matyus P., and Bathori M. (2009), C-29 Ecdysteroids from Ajuga reptans. J. Nat. Prod. 72, 929-932.

Yang L., Han H., Nakamura N., Hattori M., Wang Z., and $\mathrm{Xu} \mathrm{L.} \mathrm{(2007a),} \mathrm{Bio-guided} \mathrm{isolation} \mathrm{of} \mathrm{antioxi-}$ dants from the stems of Dendrobium auranticum var. denneanum. Phytother. Res. 21, 696-698.

Yang L., Nakamura N., Hattori M., Wang Z., Bligh S. W. A., and Xu L. (2007b), High-performance liquid chromatography-diode array detection/electrospray ionization mass spectrometry for simultaneous analysis of cis-, trans- and dihydro-2-glucosyloxycinnamic acid derivatives from Dendrobium medicinal plants. Rapid Commun. Mass Spectrom. 21, 1833-1840. 